\title{
PUBLICACIONES ELECTRÓNICAS: EXPERIENCIAS Y DESAFÍOS EN PERÚ
}

\author{
MARIA ESTHER MOGOLLÓN \\ Movimiento Amplio de Mujeres Línea Fundacional, Perú
}

\begin{abstract}
Resumen: La autora explica sus experiencias en las publicaciones electrónicas y publicaciones impresas muy importantes como la emblemática Fempress. Aborda el uso de las Tecnologías de la información y la comunicación (TIC), que han permitido compartir información y contribuido al monitoreo de acciones, propiciando la rendición de cuentas y fortalecimiento de redes de mujeres. Mediante las TICs, las mujeres han promovido alianzas y articulaciones, favoreciendo la estructuración y construcción de propuestas y consensos y relaciones más equitativas y democráticas en el movimiento feminista y de mujeres. Describe que a través de las publicaciones electrónicas, se genera e intercambia información acerca de los problemas que atraviesan las mujeres; mujeres crecen y se fortalecen, y crece de manera exponencial la información generada por ellas. Pero estas redes están integradas también por mujeres pobres, campesinas, indígenas, analfabetas, de otros idiomas o con discapacidad que no pueden acceder a las TICs y hay que buscar las formas de revertir esta situación.

Palabras clave: publicaciones electrónicas; feminismo, redes electrónicas; brechas de género.
\end{abstract}

\section{Introducción}

América Latina es una región esencialmente multicultural. Contiene a la vez fuertes desigualdades, pero paradójicamente no es el continente más pobre. Somos países dueños de grandes riquezas naturales, pero vivimos a expensas de productos importados. Las cifras de desempleo, desnutrición, morbi-mortalidad, describen la profunda desigualdad y altos niveles de disparidad social, aumentados por conflictos armados y reclamaciones sociales. Las brechas entre ricos y pobres, entre lo rural y lo urbano, entre hombres y mujeres, se han acentuado. Se reconocen, sin embargo, algunos avances y cambios.

Sólo en el Perú existen 62 etnias diferentes, con alrededor de 15 lenguajes diferentes, que no tienen posibilidades de expresarse más allá de sus comunidades culturales, algunas de ellas naciones ancestrales con una riqueza cultural que no debería perderse. El Perú atraviesa un tiempo de transformaciones, de recuperación de la democracia y de fortalecimiento institucional, después de 10 años de corrupción y violación de derechos humanos en el régimen fujimorista.

La Sociedad de la Información, definida como una sociedad mejor, más democrática, ofrece inmensas posibilidades de acortar las brechas de género existentes. Las tecnologías de la información y comunicación se vienen desarrollando de forma creciente en el país. Sin embargo, las TICs por sí mismas, no pueden crear igualdad de género ni terminar con la pobreza; son herramientas poderosas para la acción y el cambio social positivo y para 
la movilización, el intercambio de información, de conocimiento y de empoderamiento para las mujeres. Para lograr una Sociedad de la Información, democrática y con desarrollo humano, habrá que cerrar no sólo la brecha digital entre hombres y mujeres, sino también entre clases, etnias, regiones geográficas, etc.

Las organizaciones de mujeres y feministas han ido colocando la agenda de Género y TICs, lo cual les ha valido para legitimar sus esfuerzos.

\section{La Sociedad de la Información y las TICs desde un enfoque de género}

Al aplicar un enfoque de género señalamos las desigualdades y las relaciones de poder existentes entre hombres y mujeres, entre ricos y pobres, entre lo urbano y lo rural, y para nuestro trabajo lo que está conectado y lo no conectado, además de los aspectos de acceso que marcan de manera cruel las grandes dificultades actuales para acceder a las TICs.

Muchas mujeres, por razones económicas, educativas, culturales, no pueden acceder a los espacios tecnológicos y debemos buscar las formas de acortar estas brechas digitales y de acceso. ¿Cómo hacer para acortar brechas no sólo de acceso, sino también de empoderamiento?

Las mujeres peruanas, sin duda, hemos avanzado. Tenemos una legislación que contiene algunos aspectos favorables, logro de décadas de esfuerzos y demandas de las mujeres organizadas. Ha mejorado el acceso a la educación y al poder político mediante la Ley de cuotas como medida de acción afirmativa. Un sector de mujeres eleva su grado de educación a postgrados, maestrías y doctorados.

Pero las desigualdades aún existen para las mujeres peruanas y están expresadas de diversa manera. Algunas cifras ilustran esta realidad: área rural.

Hay 1.215.018 personas analfabetas y el 77. 2 \% son mujeres, la mayoría en el

Perú ocupa el segundo lugar, después de Bolivia, entre los países de América del Sur con mayores índices de mortalidad materna: 1258 muertes maternas anuales.

El $13 \%$ de las mujeres entre 15 y 19 años ya son madres con efectos nefastos sobre sus vidas.

- Se realizan 352 mil abortos por año, en su mayoría en condiciones deplorables.

- La gran mayoría de víctimas por violación son mujeres: 92.7 \%, de las cuales el $66 \%$ son menores de 14 años. Es decir, 25 mil violaciones por año, tres cada hora.

El Perú posee un rico caudal de organizaciones de mujeres que dan un soporte importante a la institucionalidad y la gobernabilidad. Actualmente un sector de ellas lucha por la Ley de Igualdad de Oportunidades con equidad de género.

\section{Publicaciones Feministas: Las experiencias}

Esta presentación está basada en la experiencia en diversas publicaciones, de las cuales guardo muchas lecciones y gratitudes. En tiempos de globalización, estas experiencias, tanto las que provienen de las publicaciones impresas como las de las publicaciones electrónicas o del uso de las TICs, dan la posibilidad de plantear lecciones aprendidas y desafíos para quienes ejercemos el periodismo feminista. 


\section{Experiencias principales:}

Publicaciones escritas (prensa plana)

- Fempress

Noticias Aliadas

Publicaciones Electrónicas:

E-Boletín Regional de Grupo Latino AWID (The Association for Women's Rights in Development -AWID).

E-Boletín Regional de RELAT (de la Red de Escritoras Latinoamericanas)

E-Boletín InfoMAMFundacional del Movimiento Amplio de Mujeres Línea Fundacional (Perú)

E-Boletín Regional Ciudadanía Sexual y web ( Del Proyecto Sexualidades, Salud y Derechos Humanos en América Latina, SSDHAL -UPCH)

\section{Las Publicaciones}

Fempress ( www.fempress.cl)

Su sede está en Chile. Red de comunicación alternativa para la Mujer en América Latina. Nació en 1981 y dejó de publicarse en el 2000, después de una evaluación profunda. Contaba con un grupo de más de 20 corresponsales en casi toda América Latina.

A la revista Fempress se la consideraba como un poderoso instrumento de intercambio que vinculaba y daba apoyo informativo a quienes trabajaban activamente por mejorar la condición de la mujer en distintos niveles y en los diferentes países de la región.

Era "la revista feminista latinoamericana por excelencia". Hacía una cobertura completa tanto del acontecer de los países desde una perspectiva de género, como del quehacer de los movimientos de mujeres y feministas de la región.

\section{Noticias Aliadas}

Agencia de Prensa con más de cuarenta años de existencia. Tiene un Boletín quincenal con información alternativa sobre América Latina con énfasis en los derechos humanos. No publica exclusivamente temas de mujer, pero en buen porcentaje son mujeres las que escriben. Se entrevista a mujeres relacionadas con la información que publican y tiene como condición transversalizar género y derechos humanos.

\section{E-publicaciones:}

E-BOLETÍN GRUPO LATINO AWID (The Association for Women's Rights in Development -AWID. www.awid.org)

Grupo Latino nace en el VIII Foro AWID como una necesidad de intercambio de las personas latinas pertenecientes a AWID. La presencia de AWID en el continente a través del e-Boletín Grupo Latino era dos veces por semana.

Se convirtió en un espacio interesante para intercambiar información acerca de los derechos humanos de la mujer, la igualdad de género y el desarrollo sostenible. Instituciones y personas de todo el continente integraron Grupo Latino AWID. Voces de escritoras, historiadoras, trabajadoras de la salud, líderes feministas, de mujeres de casi todos lados sin distinción, fueron colocadas en este espacio virtual. 
RELAT (Red de Escritoras Latinoamericanas. www.relat.org.pe ). Medio electrónico de la Red de Escritoras Latinoamericanas (RELAT). Funcionó casi durante un año.

Se crea como una de las herramientas para:

- Luchar contra la discriminación específica a las escritoras mujeres, así como para combatir los mecanismos sutiles que fomentan la censura y autocensura.

- Promover y defender la libertad de expresión.

- Difundir las obras escritas por las mujeres, y hacerlas visibles como creadoras.

Desarrollar una crítica objetiva que valore en su real dimensión la literatura escrita por mujeres en nuestra región.

InfoMAMFundacional

El Movimiento Amplio de Mujeres Línea Fundacional, iniciativa ciudadana feminista, tiene un Boletín Electrónico que se ha convertido en su medio de difusión.

La organización tiene su origen en 1996. Trabaja activamente por los derechos humanos de las mujeres, en especial por los derechos sexuales y reproductivos. Entre sus objetivos se encuentran sus esfuerzos por contribuir a una equitativa Sociedad de la Información, a la vez que promueve que el uso de las Tecnologías de la información y comunicación desde una perspectiva de género contribuyan a la reducción de la pobreza; también lucha por una sociedad inclusiva y diversa, que elimine brechas culturales, sociales, tecnológicas y económicas. Ya cuenta con experiencias y buenas prácticas del uso de las TICs a favor del movimiento de mujeres en general y rurales en particular.

Su trabajo en red se desarrolla con Mujeres de Anta (Cuzco), Piura, Ica y Lima, que reflejan sus voces, sus problemáticas, y sus demandas a través del InfoMAMFundacional. Así, también recoge sus emprendimientos y logros desde distantes lugares del país.

MAMLF realizan campañas nacionales e internacionales, se solidarizan, hacen vigilancia en salud sexual y reproductiva, y son aleccionadores sus esfuerzos como monitoradoras electrónicas de la Campaña de adhesiones nacionales e internacionales para la Ley de Igualdad de Oportunidades

Asimismo, mediante el uso de las alertas electrónicas y de uso de las TICs, desarrollaron un papel innovador a favor de la recuperación de la democracia, haciendo circular notas y materiales de campaña desde 1998 hasta el 2000, año en que cayó la dictadura. Sus empeños los ubican en la necesidad de empoderar a las mujeres campesinas quechua-hablantes de Cuzco (sierra sur del Perú), en el acceso a las TICs como medio de comunicación y difusión no sólo de sus problemas, sino de sus esfuerzos en torno a la producción agrícola, la cultura, sus talleres de capacitación y otros problemas diversos.

Mientras, la mujeres rurales de Piura (Costa norte) deciden elaborar un programa de radio que las articule a una población de 50 mil mujeres en temas de violencia contra la mujer, así como gobernabilidad y otros. Tanto mujeres urbanas como rurales integran la Red Nacional de Promotoras del MAM Línea Fundacional que lucha por el derecho a la salud y los derechos sexuales y reproductivos. A su vez, integra tanto redes nacionales como internacionales.

Integra el Grupo Latinoamericano Género \& TICs que cuenta con mayor alcance. También asume los trabajos previos realizados en Buenos Aires a través de la FLACSO y la Cátedra UNESCO sobre Género y TICs, espacio al cual pertenecen mujeres y organizaciones de la región y el MAM Línea Fundacional de Perú. 
E-Boletín Ciudadanía Sexual (www.ciudadaniasexual.org

Pertenece al proyecto: Sexualidades, Salud y Derechos Humanos en América Latina/ Universidad Peruana Cayetano Heredia. Preparado para iniciar en 2003, es bimensual.

El e-boletín busca:

Contribuir a la construcción de un marco institucional que estimule la investigación, la abogacía en salud y derechos sexuales, con énfasis en las sexualidades.

Contribuir a la articulación de investigadores y activistas que trabajan género, sexualidad y la diversidad sexual en relación con la salud y los derechos humanos.

- Impulsar el crecimiento de una comunidad interesada en temas como la diversidad sexual, las sexualidades, el erotismo, el VIH/Sida, el estado laico, los derechos sexuales y reproductivos, entre otros.

\section{Lecciones aprendidas}

El trabajo de construcción de boletines e informativos electrónicos durante varios años permite hacer algunas reflexiones y exponer lecciones aprendidas. Entre ellas:

Compartir, valorar información originada en distintas fuentes: medios de prensa y de organizaciones de mujeres, listas feministas, de VIH/SIDA y medios de prensa internacionales

Las publicaciones electrónicas son medios poderosos de intercambio de información, resultado de investigaciones y quehaceres, así como de opinión, promoviendo acciones favorables para la transformación.

Son espacios para el diálogo, el acuerdo y el disentimiento.

Nos hemos unido a Campañas Movilizativas de opinión y de solidaridades. Voces, voluntades y entusiasmo a favor de la vida y de la paz. También hemos colocado la voz de alerta sobre determinados sucesos que conmueven a los pueblos de nuestra América.

Los e-boletines contribuyen al empoderamiento de las mujeres en sus capacidades individuales, así como en contextos organizacionales y comunitarios.

Al aplicar un enfoque de género hacemos un reconocimiento de las desiguales relaciones de poder existentes entre hombres y mujeres, entre ricos y pobres, entre lo urbano y lo rural, lo conectado y lo no conectado.

Las Tecnologías de la Información y Comunicación, por sí mismas, no pueden crear igualdad de género ni terminar con la pobreza; sin embargo, son herramientas poderosas para la acción social y el cambio social positivo.

Para lograr una Sociedad de la Información, democrática y con desarrollo humano, habrá que cerrar la brecha digital, no sólo entre hombres y mujeres, sino entre clases, etnias, regiones, y otras. Esta brecha, en sí misma, es consecuencia de las desigualdades existentes.

Las TIC son una herramienta para la movilización, intercambio de información y otorgamiento de poder para las mujeres

La agenda del "género y las $\mathrm{TIC}$ " ha ido ganando legitimidad en forma constante debido al trabajo de organizaciones de mujeres y de otras organizaciones.

La Sociedad de la Información, en su definición, es una sociedad mejor, más democrática y en la que la economía del conocimiento es inherentemente equitativa, pero no hay certeza de que asegure una distribución más igualitaria de la riqueza y el poder.

También es una lección aprendida que una activista feminista y periodista pueda desenvolverse en los espacios de la Academia, contribuyendo con su labor a acortar distancias en relación a las disputas constantes entre la Academia y el activismo. Así lo 
demuestran nuestra práctica como activista del MAMLínea Fundacional y nuestra labor como responsable del Boletín Ciudadanía Sexual, del Proyecto Sexualidades, Salud y Derechos Humanos en América Latina de la Universidad Peruana Cayetano Heredia.

\section{Perspectivas}

Gloria Bonder, en sus textos sobre "Las nuevas tecnologías de información y las mujeres: reflexiones necesarias"', hace una revisión de muchas páginas de Internet dedicadas a las mujeres, así como de las producidas por las mismas organizaciones. De acuerdo a ello hace una serie de propuestas al respecto, a las que unimos las que traemos desde la experiencia propia, así:

Promover el desarrollo de las mujeres, brindándoles recursos para acceder y utilizar nueva información y tecnologías de comunicación.

Las TIC ofrecen inmensas posibilidades de reducir la pobreza, superar el aislamiento de las mujeres, darles voz, mejorando la gobernanza y avanzando en la igualdad de género.

Promover la conciencia de las aplicaciones organizacionales de las TIC, por ejemplo, para la investigación, para el trabajo en redes, para hacer lobby y conferenciar, y para demostrar el rol que pueden jugar las TIC en el avance de la igualdad de género.

La Sociedad de la Información debe contener las necesidades de las mujeres, que son más del $50 \%$ de la población mundial. Debe contribuir al empoderamiento de mujeres.

En este tiempo de globalización y de las TICs, en un mundo en permanente cambio, la información es abundante, rápida, descartable; las/los periodistas vamos atrás, atrás del hecho, del tiempo, y muchas veces somos superficiales, sin tiempo para hacer una lectura crítica de los hechos. No podemos abdicar de ese análisis, de la investigación².

Existe una tendencia a la uniformización de títulos y de contenidos: "una manera de neutralizar la información". La idea sería ser más creativas, y aplicar nuestra mirada.

La calidad no puede ceder a la cantidad.

Es una comunicación más igualitaria que la presencial, lo cual favorece a las mujeres y a otros grupos marginados. Entonces, hay que pensar nuevas estrategias educativas, políticas, institucionales, que contengan la igualdad de oportunidades de hombres y mujeres en Internet, más allá del acceso a la computadora y el Internet.

Prepararnos para abordar temas cuya información estará en manos de pocos: biotecnología, clonación, Genoma humano... entre otros. ¿Cómo salir del círculo de lo que mandan los grandes medios? ¿Cómo dejar de ser simples espectadoras de lo que está pasando, esperando a que nos den la información a medias? Transformar la información complicada en debatible, entendible para la ciudadanía, es una de las responsabilidades; la otra gran tarea es abordar la manera cómo nos afectan, o no, estas tecnologías, y también, como se alejan de nuestro acceso.

Organizar, participar en eventos donde múltiples voces opinan sobre complicados temas, contribuye a la democratización de la información. Nuestra mirada contribuirá a develar o promover aquello positivo para las mujeres.

Mirar más lo local, publicar lo local, y lo global. Buscar un equilibrio.

Profundizar, provocar el debate de las teorías feministas junto a temas que procuran una mejor comprensión de las diversidades sexuales, culturales, de los cambios.

Apropiarnos de las tecnologías y las posibilidades que ofrecen para la transformación de la sociedad. 
Nos falta:

Ser más inclusivas. Incluir voces e informaciones de la diversidad geográfica, sexual y cultural.

Promover mayor participación en la discusión. No queremos ser sólo nosotras las que estimulemos el debate.

Sabemos que quienes accedemos a las TICs y a los espacios virtuales somos aún muy pocas personas.

No hay cifras ni indicadores que permitan (en Perú) evaluar las carencias o impactos de nuestra información. Hay que estimular estas investigaciones.

Muchas mujeres, por razones económicas, educativas o culturales, no pueden acceder a estos espacios tecnológicos y debemos buscar las formas de acortar estas brechas.

En los países andinos quechua-hablantes (Bolivia, Perú y Ecuador) se hace más patente el centralismo con su secuela de segregación y aislamiento, donde se menosprecia e ignora sistemáticamente la tradición oral en quechua y castellano.

Olvidamos con frecuencia que la América Latina es una región esencialmente multicultural: sólo en el Perú existen 62 etnias diferentes, con alrededor de 15 lenguajes diferentes, que no tienen posibilidades de expresarse más allá de sus comunidades culturales, algunas de ellas naciones ancestrales con una riqueza cultural que no debería perderse.

¿Cómo hacer para conservar esta riqueza y a la vez acortar brechas no sólo de acceso, sino también de empoderamiento?

¿Hasta qué punto el uso de los medios electrónicos, del Internet, logra mejorar la posición social, las oportunidades laborales, la participación de las mujeres? Tanto por aspectos teóricos, como por sus implicancias prácticas y políticas, es bueno investigar la comprensión de las experiencias de las Mujeres en la red.

¿En qué medida ellas reiteran en sus interacciones la marca de los patrones de género que circulan en el mundo off line? Este nuevo escenario ¿contiene la potencialidad de revertir o al menos cuestionar las categorías de género y el rol que ellas pueden desarrollar en él?

La comunicación on line, ¿es horizontal, hay un menor control? ¿Qué significa la invisibilización de los cuerpos, que pasa con las identidades?

Aunque con muchas trabas y dificultades, el Perú avanza en su proceso de democratización y de construcción de la Sociedad de la Información, pero ésta sólo será posible con el acortamiento de todo tipo de brechas que no sólo signifiquen el acceso al uso de las TICs, sino que permitan también la participación de las mujeres en el diseño de las políticas.

Las actuales autoridades no están tomando en cuenta los compromisos internacionales de carácter vinculante que ha realizado el Estado Peruano: la Convención sobre la Eliminación de todas las Formas de Discriminación contra la Mujer (CEDAW), la Convención Interamericana para Prevenir, Sancionar y Erradicar la violencia contra la mujer, por ejemplo, o la Plataforma de Acción de Beijing y el Programa de Acción de la Conferencia Internacional de Población y Desarrollo (El Cairo). Asimismo, el cumplimiento de los Objetivos del Milenio al 2014. Hacen falta investigaciones con un enfoque de género que reflejen las grandes brechas existentes que constituyen un freno para el avance de las mujeres.

Se requieren Leyes y políticas públicas integrales, recursos que promuevan la igualdad de oportunidades con equidad de género y con medidas de acción afirmativa 
temporales que mejoren el acceso y la apropiación de las TICs por parte de las mujeres, como una forma de mejorar sus condiciones de vida y su posición en la sociedad.

Es crucial que, tanto las organizaciones de mujeres como las personas que trabajan por la igualdad del género, se eduquen sobre los temas de las políticas de TIC y se involucren en el cabildeo para la formulación de políticas en sus países y regiones.

La innovación en la Sociedad de la Información no es sólo una palabra, sino la acción aplicada a la educación y a otros campos de la vida que contribuya al acortamiento de las brechas de género, de clase, geográficas y otras, permitiendo un mejor ejercicio de la ciudadanía de las personas. La innovación no sólo debe enfocar los aspectos tecnológicos, sino también promover la creación de programas que contribuyan a cambiar la imagen desvalorizada de la mujer y a mejorar sus condiciones de vida.

Abrir, mejorar el diálogo entre el Estado, Sociedad Civil, Empresa y Academia, donde se tome en cuenta la voz de las mujeres, lo cual permitirá un mejor camino hacia la Sociedad de la Información.

\section{Notas}

Copyright (C) 2004 by Revista Estudos Feministas.

1 Gloria BONDER, 2001

2 Laura GREENHALG, 2002.

\section{Fuentes}

BONDER, Gloria. Las nuevas tecnologías de información y las mujeres: Reflexiones necesarias. Reunión de Expertos sobre Globalización, Cambio Tecnológico y Equidad de Género. Sao Paulo, Brasil, 5 y 6 de noviembre de 2001. CEPAL-GTZ "Institucionalización del enfoque de género en la CEPAL y Ministerios Sectoriales" de la Unidad Mujer y Desarrollo de la Comisión Económica para América Latina y el Caribe (CEPAL). 2001. (Versión preliminar).

CALANDRIA. Internet, sus servicios más usados y experiencias de sitios Webs internacionales de participación ciudadana. Proyecto del Portal de la Sociedad Civil del Centro de Investigación ACS. Lima, Perú, 2002

CLADEM Perú. Diagnóstico sobre la situación de los derechos sexuales y reproductivos: 1995-2003. 2003

GREENHALGH, Laura. "Precursores o retardatários?". In: COMISSÃO DE CIDADANIA E REPRODUÇÃO. Olhar sobre a Mídia. Belo Horizonte: Mazza Edições Ltda., 2002.

Informe. Taller de trabajo: "Genero y TICs en América Latina: Cumbre Mundial sobre la Sociedad de la Información y mas allá". FLACSO/ Cátedra Regional UNESCO, Mujer, Ciencia y Tecnología en América Latina. Octubre. Bs. Aires, Argentina, 2003

MOGOLLÓN, María Esther."Género \& TICs". Ponencia en TALLER DE TRABAJO. GÉNERO \& TICS. Concytec. Lima, Perú, 2003

MOGOLLÓN, María Esther. "Sociedad de la Información, Género \& TICs". Ponencia en CAUCUS DE GÉNERO. CUMBRE MUNDIAL DE LA SOCIEDAD DE LA INFORMACIÓN. Ginebra, Suiza, 2003.

MUJERES por la IGUALDAD de OPORTUNIDADES. Cartilla para la Incidencia. Por una Ley de Igualdad de Oportunidades con equidad de Género. Perú, 2003

SANTOYO Cadena, Eduardo. "En camino hacia la Sociedad de la Información". Ponencia para el Ministerio de Transportes y Comunicaciones. Red Científica Peruana, Lima, Perú, 2003. 
Electronic Publications: Experiences and Challenges in Peru

Abstract: The author explains her experiences on the electronic publication field, and in very important printed publications such as the emblematic Fempress. She approaches the use of the Technologies of information and communication (TIC), that have allowed to share information and contributed to the invigoration and monitoring of actions that propitiate the rendering of information and strengthening of women's nets. By using the TICs, women have promoted alliances and articulations, favoring the structuring and construction of proposals, consents and more equal and democratic relationships in the feminist and women movement. The author describes that through the eletronic publications it is generated and exchanged information about the problems that women live; their efforts and advances are exposed, the solidarity is developed, and a great variety of themes is approached. The nets of women grow and get strengthen, and the information generated by them, grows in an exponential way. But these nets are also integrated by poor women, peasants, indigenous, illiterate, women who speak other languages or with discapacity, who cannot access the TICs, so it is necessary to look for the ways of reverting this situation.

Key-words: electronic publications, feminism, electronic nets, gender breach. 